\title{
Immune Responses Following Mouse Peripheral Nerve Xenotransplantation in Rats
}

\author{
Lai-Jin Lu, ${ }^{1}$ Jia-Bing Sun, ${ }^{1,2}$ Zhi-Gang Liu, ${ }^{1}$ Xu Gong, ${ }^{1}$ Jian-Li Cui, ${ }^{1}$ and Xi-Guang Sun ${ }^{1}$ \\ ${ }^{1}$ Department of Hand Surgery, First Hospital of Jilin University, Changchun, Jilin 130021, China \\ ${ }^{2}$ Department of Orthopedics, First Clinical College of Harbin Medical University, Harbin, \\ Heilongjiang 150001, China \\ Correspondence should be addressed to Jia-Bing Sun, sunjiabing@live.cn
}

Received 12 May 2009; Accepted 24 July 2009

Recommended by Xudong Huang

Xenotransplantation offers a potentially unlimited source for tissues and organs for transplantation, but the strong xenoimmune responses pose a major obstacle to its application in the clinic. In this study, we investigate the rejection of mouse peripheral nerve xenografts in rats. Severe intragraft mononuclear cell infiltration, graft distension, and necrosis were detected in the recipients as early as 2 weeks after mouse nerve xenotransplantation. The number of axons in xenografts reduced progressively and became almost undetectable at week 8 . However, mouse nerve xenotransplantation only led to a transient and moderate increase in the production of Th1 cytokines, including IL-2, IFN- $\gamma$, and TNF- $\alpha$. The data implicate that cellular immune responses play a critical role in nerve xenograft rejection but that further identification of the major effector cells mediating the rejection is required for developing effective means to prevent peripheral nerve xenograft rejection.

Copyright ( 2009 Lai-Jin Lu et al. This is an open access article distributed under the Creative Commons Attribution License, which permits unrestricted use, distribution, and reproduction in any medium, provided the original work is properly cited.

\section{Introduction}

Because peripheral nerve tissue has very limited spontaneous regenerative capacity, the development of strategies to facilitate axonal regeneration is highly important for treatment of peripheral nerve injuries [1-3]. Although autotransplantation is considered the "gold standard" approach for bridging nerve gaps, autogenous grafts provide only a limited source of graft material and graft harvesting may result in secondary site morbidity. Allogeneic and xenogeneic nerve allografts provide an attractive alternative for patients, for whom the use of autograft is infeasible or undesirable. However, immunological rejection poses a major obstacle to clinical allogeneic and xenogeneic transplantation.

Unlike organ transplantation, in most cases peripheral nerve injury is not life-threatening, and therefore, peripheral nerve xenotransplantation can only be considered when its benefit is weighted against the risk associated with the therapy, such as the side effects of immunosuppressive drugs. Peripheral nerves contain Schwann cells, fibroblasts, and extracellular matrix. Schwann cells and fibroblasts both express MHC antigens and play an important role in eliciting immunological rejection after allogeneic nerve transplantation [4]. However, immunologic responses following nerve xenotransplantation are relatively poorly understood. In this study, we investigate the immunological response and graft survival in rats after nerve xenotransplantation from mice. We show that mouse nerve xenografts were vigorously rejected in rat recipients, and that the rejection was associated with severe intragraft infiltration by mononuclear cells despite only a transient and moderate increase in Th1 cytokines detected in the recipients.

\section{Materials and Methods}

2.1. Animals. Female SD rats (weighed between 150 and $200 \mathrm{~g}$ ) and adult female BALB/c mice were used as the recipients and donors, respectively. All animals were purchased from the Second Affiliated Hospital of Harbin Medical University (Haerbin, China). All surgical procedures and postoperative care of the animals were approved by the Institutional Animal Ethics Committee. 


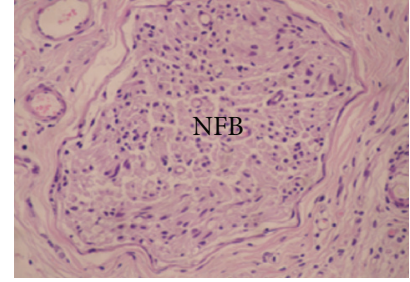

(a)

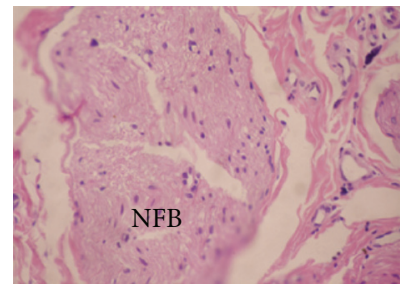

(c)

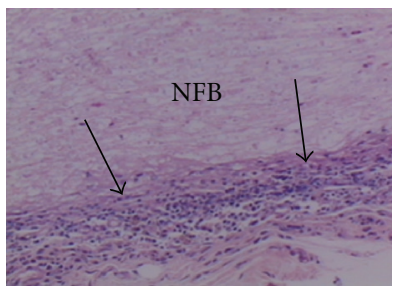

(e)

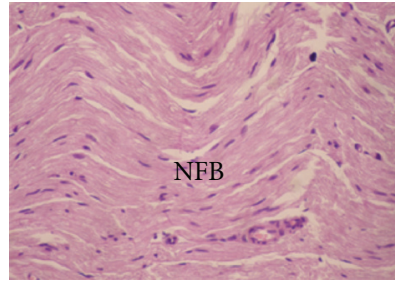

(b)

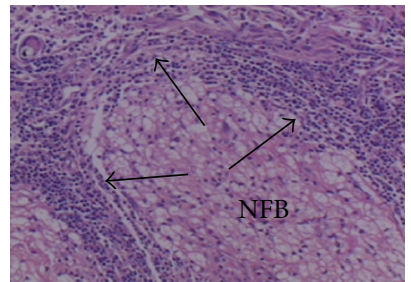

(d)

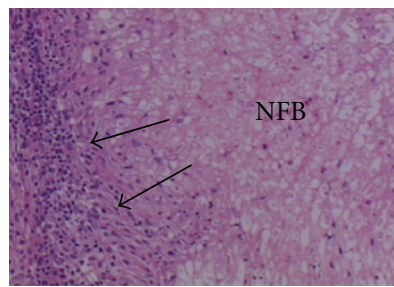

(f)
Figure 1: Severe mononuclear cell infiltration and graft destruction in mouse nerve xenografts. Autologous (a)-(c) and xenogeneic mouse (d)-(f) nerve grafts were harvested at weeks 2 (a) and (d), 4 (b) and (e), and 8 (c) and (f) posttransplantation and analyzed by light microscopy with $\mathrm{H} \& \mathrm{E}$ staining. Five rats per group were examined at each time point, and representative samples $(x 40)$ are shown. Mononuclear cell infiltration was detected (arrowheads) around nerve fiber bundle (NFB) in mouse nerve xenografts at all time points examined.

2.2. Peripheral Nerve Xenotransplantation. All surgical procedures were performed under anesthesia with ketamine $(50 \mathrm{mg} / \mathrm{kg}$; i.m.). To prepare donor nerve grafts, the mouse sciatic nerve was exposed through a dorsal gluteal muscle splitting incision, and a segment $(1 \mathrm{~cm})$ of sciatic nerve was harvested and used immediately. The skin over the recipient right hindlimb was incised, and the muscle was bluntly dissected to expose the superficial peroneal nerve, and a $1 \mathrm{~cm}$ gap was created. The mouse sciatic nerve graft was interposed to the transected nerve and immediately repaired with 10-0 nylon epineurial sutures. For autograft recipients, the right superficial peroneal nerve was exposed in an identical fashion, transected with microscissors, and tensionfree repair of the nerve gap $(1 \mathrm{~cm})$ was then performed with 10-0 nylon epineurial sutures.

2.3. ELISA. Sera were collected from recipient mice at various times, and the levels of IL-2, IL-4, IFN- $\gamma$, and TNF- $\alpha$ were determined using ELISA kits (Westang Biotechnology, Shanghai, China) according to the manufacturer's instructions.

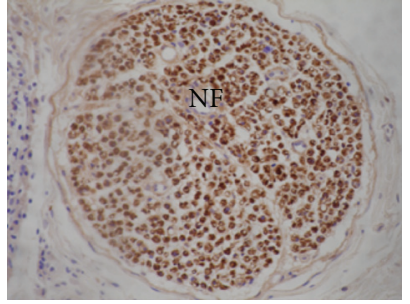

(a)

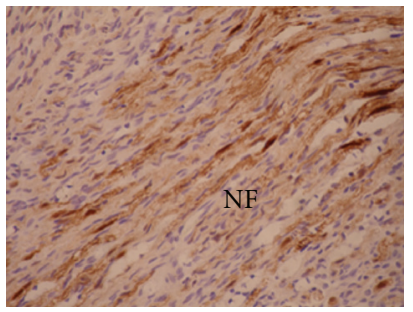

(c)

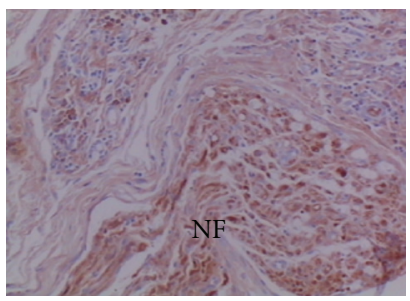

(e)

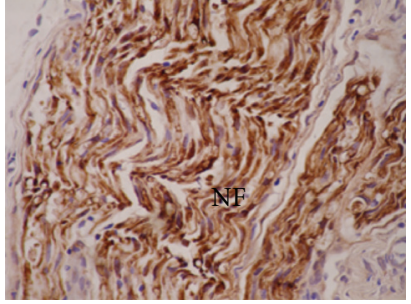

(b)

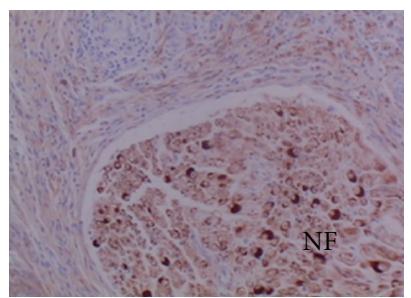

(d)

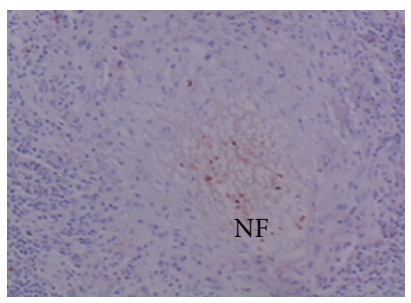

(f)
FIGURE 2: Rapid and progressive loss of axons in mouse nerve xenografts. Autologous (a)-(c) and xenogeneic mouse (d)-(f) nerve grafts were harvested at weeks 2 (a) and (d), 4 (b) and (e), and 8 (c) and (f) posttransplantation and analyzed by immunohistochemistry with S-100 staining. Five rats per group were examined at each time point (these are the same animals shown in Figure 1), and representative samples $(x 40)$ are shown. NF, nerve fibers.

2.4. Histological Evaluation. Animals were sacrificed at various time points after transplantation, and the nerve grafts were harvested and cut in half horizontally. One segment was immersed in a $10 \%$ formaldehyde solution, dehydrated in ethanol, and embedded in paraffin. The paraffin nerve sections were stained with hematoxylin and eosin (H\&E) and by immunohistochemistry for S-100. Briefly, sections for S-100 staining were first incubated with polyclonal rabbit anti-S-100 antibodies (Westang Biotechnology), washed, and followed by staining with biotinylated goat antirabbit antibodies (Westang Biotechnology). DAB Substrate was used for colorimetric detection. Another segment of the nerve graft was immersed in a 3\% cold gluteraldehyde solution, postfixed with osmium tetroxide, dehydrated in ethanol, and embedded in Araldite 502. Ultrathin sections were obtained with an ultramicrotome (ULTRACUT E, Reichert-Jung, Vienna, Austria), stained with lead citrate and uranyl acetate, and observed under a JEOL transmission electron microscope (JEM-1220; JEOL Ltd., Tokyo, Japan). 
2.5. Statistical Analysis. Differences between group means were tested using Student's $t$-test by Microsoft Excel software. A $P$-value of $<.05$ was considered to be significant.

\section{Results}

Recipient rats were sacrificed 2, 4, and 8 weeks after transplantation and the nerve grafts were examined macroscopically and histologically $(n=5$ per group at each time point). All autografts showed no distension or adhesion to the ambient tissues throughout the 8-week observation period. However, xenografts removed at week 2 markedly dilated and adhered to the surrounding tissues. While some xenografts showed moderately reduced distension at week 4, graft adhesion to the ambient tissues was progressively overwhelming in all animals during the 8-week observation period.

Histological examination at weeks 2 and 4 revealed very mild mononuclear cell infiltration with otherwise normal peripheral nerve structure in autografts (Figures $1(\mathrm{a})-1(\mathrm{~b})$ and 2(a)-2(b)). However, most autografts showed signs of Wallerian degeneration at week 8 , with moderate axonal degeneration and nerve fiber fractions (Figures 1(c) and 2 (c)). Consistent with the light microscopic analysis, electron microscopy confirmed the preservation of normal fascicular structure in autografts at weeks 2 and 4 (Figures 3(a)3(b)), but Schwann cell cytoplasmic degeneration, chromatin condensation, and nuclear collapse were identified in the autografts at week 8 (Figure 3(c)).

Graft rejection was identified in all xenografts. Severe mononuclear cell infiltration, distension and necrosis were detected in all xenografts as early as 2 weeks after transplantation (Figures 1(d)-1(f)). The number of axons in the grafts markedly reduced by week 2 and became almost undetectable at week 8 (Figures 2(d)-2(e)). Electron microscopy revealed inflammatory infiltrates in nerve fibers, Schwann cell nuclear pycnosis and mitochondrial damage, and progressive destruction and loss of myelinated fibers in all xenografts (Figures 3(d)-2(f)).

Sera were collected from xenograft recipients prior to 2,4 , and 8 weeks after transplantation, and the levels of IL-2, IL- 4 , IFN- $\gamma$, and TNF- $\alpha$ were determined by ELISA (Table 1). While the serum levels of IL-2, IFN- $\gamma$, and TNF- $\alpha$ at week 2 were statistically higher in the xenograft recipients than in the control rats, these increases were relatively less marked and transient. The serum levels of all these cytokines in the xenograft recipients returned to normal by week 4 after transplantation.

\section{Discussion}

Xenotransplantation provides a possible solution to the severe shortage of allogeneic donors that limits clinical transplantation [5-7], but the virulent xenoimmune responses pose a strong barrier to clinical xenotransplantation [7]. Transplantation of discordant xenografts results in hyperacute rejection due to the presence of natural antibodies (NAbs) in the recipient sera. The major NAbs that mediate

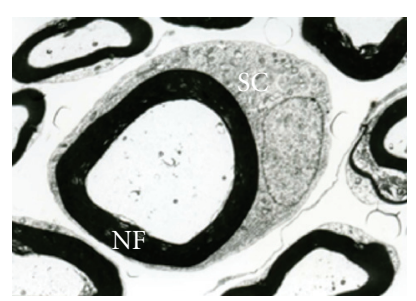

(a)

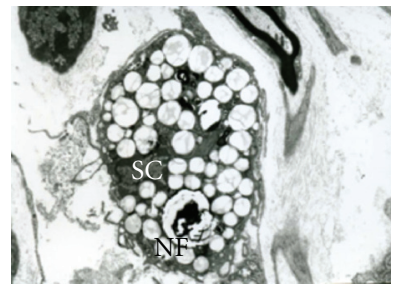

(c)

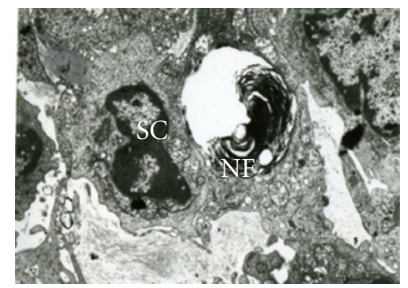

(e)

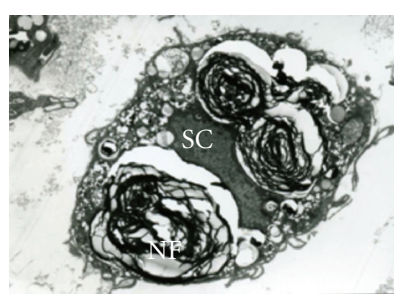

(b)

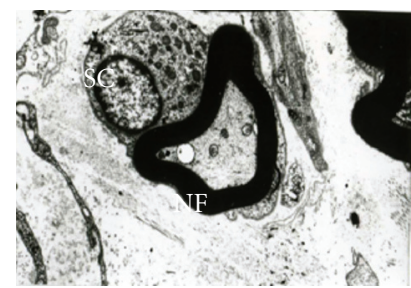

(d)

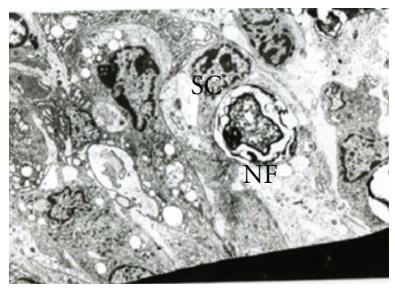

(f)
FIGURE 3: Electron microscopic analysis of autologous and xenogeneic nerve grafts. Autologous (a)-(c) and xenogeneic mouse (d)(f) nerve grafts were examined at weeks 2 (a) and (d), 4 (b) and (e), and 8 (c) and (f) posttransplantation. Five rats per group were analyzed at each time point (these are the same animals shown in Figure 1$)$, and representative samples $(x 5,000)$ are shown. SC, Schwann cell; NF: nerve fibers.

hyperacute rejection are those which recognize the $\alpha 1,3 \mathrm{Gal}$ antigens $[8,9]$. However, anti- $\alpha 1,3 \mathrm{Gal}$ Abs are unlikely to mediate the rejection of nerve xenografts, as neural cells do not express $\alpha 1,3 \mathrm{Gal}$ [10]. Thus, cellular immune responses, which play a critical role in nerve allograft rejection [11], are likely to be more important than humoral immune responses in nerve xenograft rejection. Indeed, nerve xenograft rejection has been relatively poorly studied compared to other cellular or organ xenografts. Here, we observed that mouse nerve xenografts can be vigorously rejected in rats, and the rejection is associated with a severe mononuclear cell infiltration in the grafts.

Schwann cells are critical for nerve regeneration, but also the major cells trigger the rejection of nerve allografts because of their MHC expression. It has been reported that cold preservation can decrease the number of Schwann cells [12] and reduce the immunogenicity of nerve allografts $[13,14]$. Although recipient Schwann cells may migrate into the graft and support axon regeneration, donor Schwann cells in the graft are required for the regeneration of a long nerve graft [15]. Given the possibility that the recipient Schwann cells may be less efficient in migrating into nerve xenografts than into allografts, cold preservation could have 
TABLE 1: Serum levels of cytokines in xenograft recipients. Eight animals were analyzed at each time point and data are presented as mean $( \pm$ SDs).

\begin{tabular}{lcccc}
\hline Group & IL-2 $(\mathrm{pg} / \mathrm{mL})$ & $\mathrm{IL}-4(\mathrm{pg} / \mathrm{mL})$ & $\mathrm{INF}-\gamma(\mathrm{pg} / \mathrm{mL})$ & $\mathrm{TNF}-\alpha(\mathrm{pg} / \mathrm{mL})$ \\
\hline Control & $15.66 \pm 1.26$ & $2.04 \pm 0.27$ & $12.41 \pm 1.11$ & $13.81 \pm 3.28$ \\
Xenografted Week 2 & $20.04 \pm 1.65^{*}$ & $1.42 \pm 0.17$ & $22.74 \pm 2.45^{*}$ & $24.58 \pm 2.53^{*}$ \\
Week 4 & $16.14 \pm 1.91$ & $1.92 \pm 0.24$ & $14.53 \pm 2.3$ & $15.98 \pm 1.75$ \\
Week 8 & $13.9 \pm 4.25$ & $2.18 \pm 0.2$ & $12.09 \pm 2.55$ & $14.07 \pm 1.95$ \\
\hline
\end{tabular}

${ }^{*} P<.05$ compared to controls.

an even greater limitation for preventing nerve xenograft rejection. Although histological analysis confirmed severe mononuclear cell infiltration and complete nerve xenograft rejection, the recipient rats showed only a transient and moderate increase in Th1 cytokine production. Further studies are needed to determine whether peripheral nerve xenografts are less efficient than other types of xenografts in eliciting the systemic $\mathrm{T}$ cell immune responses. It is possible that other effector cells, such as Th17 cells, NK cells, and macrophages, may play an important role in the rejection of mouse nerve xenografts in rats. Previous studies have shown that NK cells and macrophages contribute significantly to xenograft rejection due to the genetic incompatibility in MHC class I [16] and CD47 [17, 18], which are needed for inhibiting NK cell and macrophage activation, respectively. Further studies are clearly needed to identify the major effector cells in the rejection of peripheral nerve xenografts.

\section{Acknowledgments}

This work was supported by a Grant from the First Hospital of Jilin University and Jilin Provincial Science \& Technology Department (no. 20070729-3).

\section{References}

[1] B. S. Bregman, "Regeneration in the spinal cord," Current Opinion in Neurobiology, vol. 8, no. 6, pp. 800-807, 1998.

[2] N. K. Loh, S. Woerly, S. M. Bunt, S. D. Wilton, and A. R. Harvey, "The regrowth of axons within tissue defects in the cns is promoted by implanted hydrogel matrices that contain BDNF and CNTF producing fibroblasts," Experimental Neurology, vol. 170, no. 1, pp. 72-84, 2001.

[3] J.-H. Ye and J. D. Houle, "Treatment of the chronically injured spinal cord with neurotrophic factors can promote axonal regeneration from supraspinal neurons," Experimental Neurology, vol. 143, no. 1, pp. 70-81, 1997.

[4] J. M. Rovak, D. K. Bishop, L. K. Boxer, S. C. Wood, A. K. Mungara, and P. S. Cederna, "Peripheral nerve transplantation: the role of chemical acellularization in eliminating allograft antigenicity," Journal of Reconstructive Microsurgery, vol. 21, no. 3, pp. 207-213, 2005.

[5] J. L. Platt, "Immunobiology of xenotransplantation," Transplant International, vol. 13, supplement 1, pp. S7-S10, 2000.

[6] D. K. C. Cooper, B. Gollackner, and D. H. Sachs, "Will the pig solve the transplantation backlog?" Annual Review of Medicine, vol. 53, pp. 133-147, 2002.
[7] Y.-G. Yang and M. Sykes, "Xenotransplantation: current status and a perspective on the future," Nature Reviews Immunology, vol. 7, no. 7, pp. 519-531, 2007.

[8] K. Yamada, K. Yazawa, A. Shimizu, et al., "Marked prolongation of porcine renal xenograft survival in baboons through the use of [alpha]1,3-galactosyltransferase geneknockout donors and the cotransplantation of vascularized thymic tissue," Nature Medicine, vol. 11, no. 1, pp. 32-34, 2005.

[9] K. Kuwaki, Y.-L. Tseng, F. J. M. F. Dor, et al., "Heart transplantation in baboons using [alpha] 1,3-galactosyltransferase geneknockout pigs as donors: initial experience," Nature Medicine, vol. 11, no. 1, pp. 29-31, 2005.

[10] S. Magnusson, V. Strokan, L. Svensson, J.-E. Månsson, L. Rydberg, and M. E. Breimer, "Expression of carbohydrate xenoantigens on porcine peripheral nerve," Xenotransplantation, vol. 12, no. 1, pp. 49-58, 2005.

[11] I. K. Fox, A. Jaramillo, D. A. Hunter, S. R. Rickman, T. Mohanakumar, and S. E. Mackinnon, "Prolonged coldpreservation of nerve allografts," Muscle \& Nerve, vol. 31, no. 1, pp. 59-69, 2005.

[12] A. D. Levi, P. J. Evans, S. E. Mackinnon, and R. P. Bunge, “Cold storage of peripheral nerves: an in vitro assay of cell viability and function," Glia, vol. 10, no. 2, pp. 121-131, 1994.

[13] G. M. Hare, P. J. Evans, S. E. Mackinnon, et al., "Effect of cold preservation on lymphocyte migration into peripheral nerve allografts in sheep," Transplantation, vol. 56, no. 1, pp. 154162, 1993.

[14] I. K. Fox, A. Jaramillo, D. A. Hunter, S. R. Rickman, T. Mohanakumar, and S. E. Mackinnon, "Prolonged coldpreservation of nerve allografts," Muscle \& Nerve, vol. 31, no. 1, pp. 59-69, 2005.

[15] S. Hall, "Axonal regeneration through acellular muscle grafts," Journal of Anatomy, vol. 190, part 1, pp. 57-71, 1997.

[16] J. D. Seebach, C. Comrack, S. Germana, C. LeGuern, D. H. Sachs, and H. DerSimonian, "HLA-Cw3 expression on porcine endothelial cells protects against xenogeneic cytotoxicity mediated by a subset of human NK cells," Journal of Immunology, vol. 159, no. 7, pp. 3655-3661, 1997.

[17] H. Wang, J. Verhalen, M. L. Madariaga, et al., "Attenuation of phagocytosis of xenogeneic cells by manipulating CD47," Blood, vol. 109, no. 2, pp. 836-842, 2007.

[18] K. Ide, H. Wang, J. Liu, et al., "Role for CD47-SIRP $\alpha$ signaling in xenograft rejection by macrophages," Proceedings of the National Academy of Sciences of the United States of America, vol. 104, no. 12, pp. 5062-5066, 2007. 


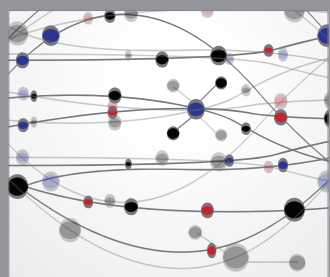

The Scientific World Journal
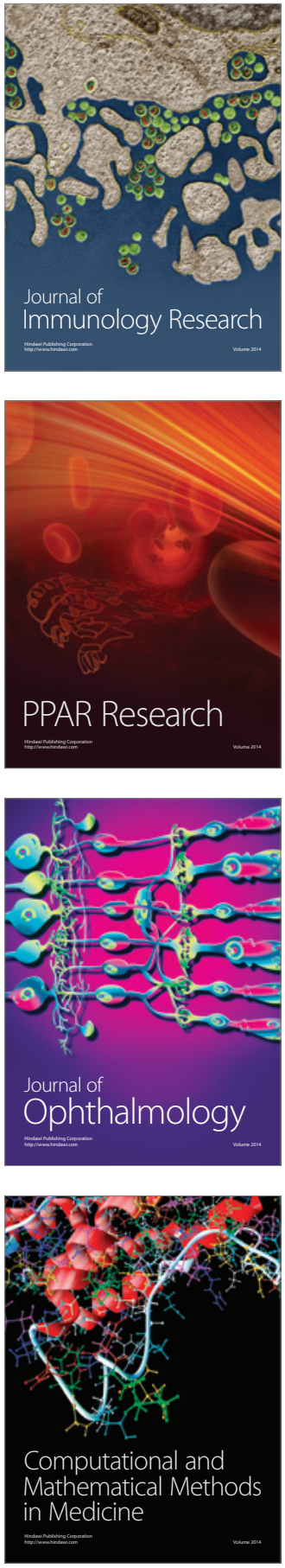

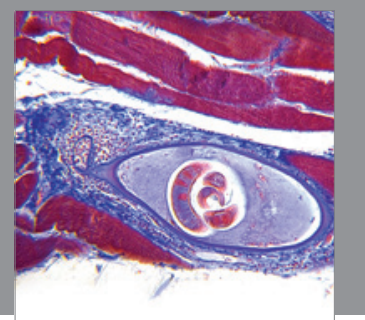

Gastroenterology

Research and Practice
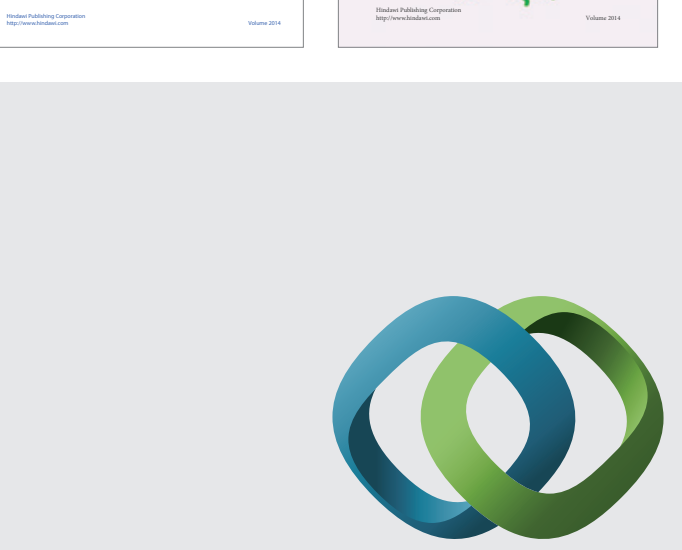

\section{Hindawi}

Submit your manuscripts at

http://www.hindawi.com
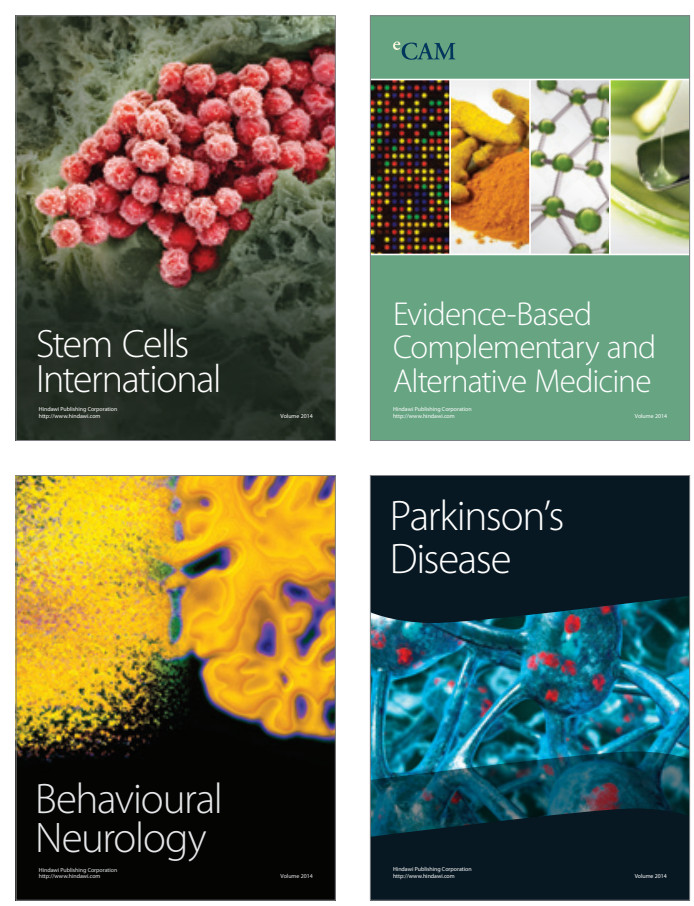

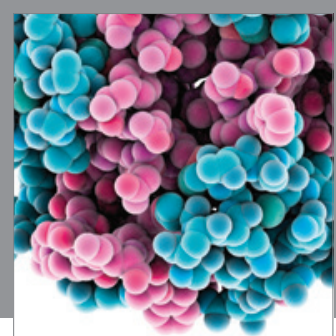

Journal of
Diabetes Research

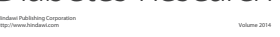

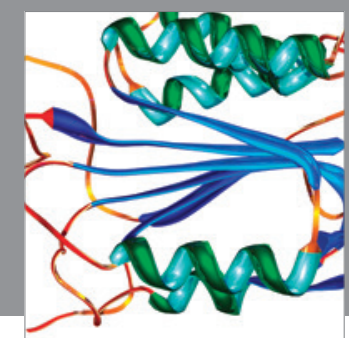

Disease Markers
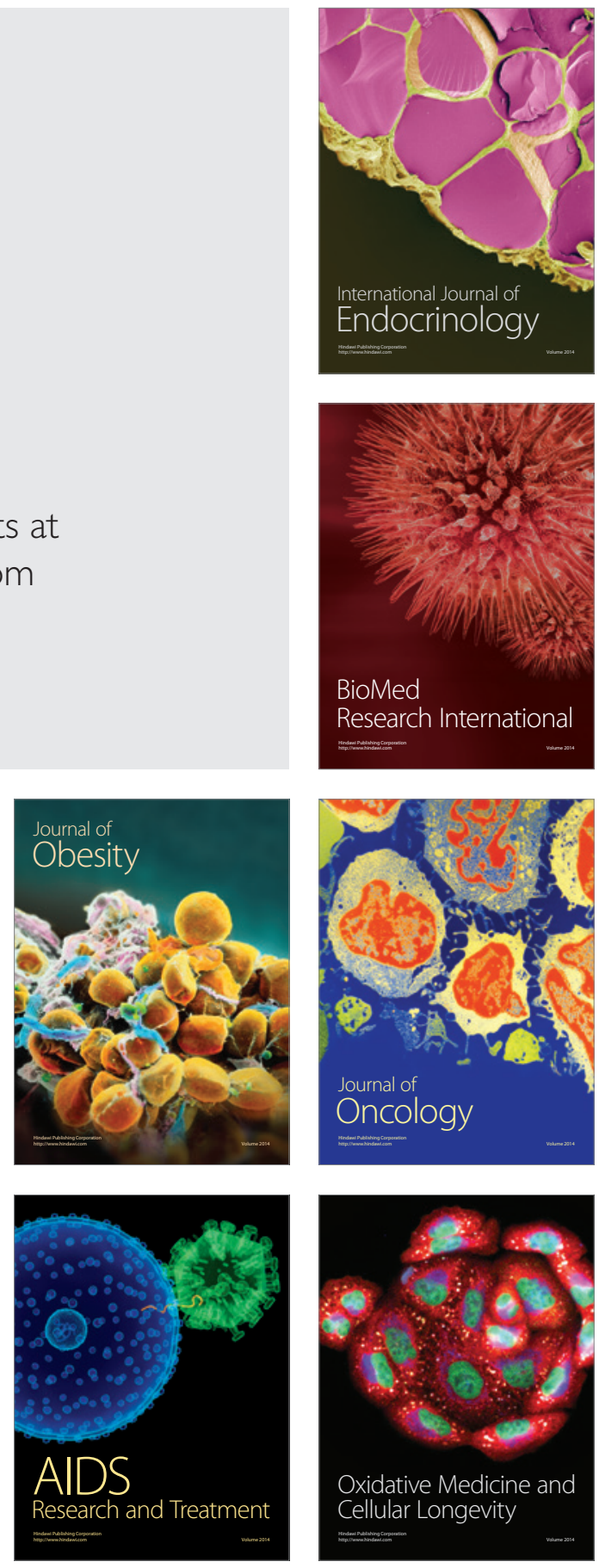